\title{
Residual differential brightness thresholds following removal of visual neocortex in rats
}

\author{
T. E. LeVERE and JEANNE MILLS \\ Neuropsychology Laboratory, N. C. State University, Raleigh, North Carolina 27607
}

\begin{abstract}
The present research is concerned with whether the differential brightness threshold in rats is changed by ablation of posterior neocortex. The results indicate that, while posterior decortication can have persistent effects on overt choice performance, the lesion apparently has little effect upon the rat's psychophysical threshold.
\end{abstract}

From the early systematic researches of Lashley (1935), it is apparent that posterior decortication in the rat severely disrupts a preoperatively acquired brightness discrimination. However, the ability to differentiate brightness difference is not beyond the capabilities of the destriate rat, since the behavior may be reinstated with postoperative discrimination training. Moreover, it has recently been shown that this postoperative recovery of function is not independent of the originally learned brightness discrimination, since animals postoperatively retrained on the reversal of the preoperative discrimination are severely impaired relative to animals postoperatively retrained on the original preoperative discrimination (LeVere \& Morlock, 1973, 1974). Thus, while visual decortication may certainly disrupt the performance of a learned brightness discrimination, apparently much of the preoperatively learned behavior is just as certainly spared.

Given that certain associations concerned with a brightness habit (engrams) may be unaffected by destruction of striate cortex in rat, a question of some importance is whether the rat's psychophysical response to light is also unaffected. An early indication that there might be a substantial difference in the rat's psychophysical response to light following lesions of the visual cortex stems from Lashley's (1930) observation that visual decortication produces a twofold increase in the differential brightness threshold. On the other hand, more recent data offered by Cooper, Freeman, and Pinel (1967) suggest that if a generalized performance deficit is accounted for, then the absolute brightness threshold is virtually unchanged following striate lesions in rat.

This research was supported by PHS Grant NS12459 to T. E. LeVere. The authors wish to thank L. Gonder for her help in preparing this report. Requests for reprints should be addressed to: T. E. LeVere, Neuropsychology Laboratory, Department of Psychology, North Carolina State University, Raleigh, North Carolina 27607.
Lashley, it must be noted, also reported a generalized performance deficit in rats with lesions of the visual cortex, but did not consider this when determining their differential brightness threshold. If it is valid to ignore the fact that the brain-damaged rat may commit errors not directly a function of a change in threshold and thus accept Lashley's conclusion, then the visual neocortex is apparently critical to the detection of differences in brightness but not absolute quantities of light. This implied involvement of visual cortex in differential, but not absolute, brightness would be an important finding concerning brain-behavior relationships should it be true.

But Lashley's conclusion may be suspect on grounds other than the observed generalized performance deficit. Principally, the problem centers on the sensitivity of the procedure he used to estimate the differential brightness threshold of normal rats and of rats with lesions of the visual cortex. Basically, this involved a series of discrimination problems, with progressively smaller brightness differences. A rat progressed through this series of problems until failing to reach criterion, at which time training was stopped and the brightness difference of the just failed problem was assumed to be below the animal's differential brightness threshold. Following visual decortication, rats never progressed as far through the series of discrimination problems as they did when normal, suggesting to Lashley that the striate lesion produced an increase in the differential brightness threshold. The problem is, however, that the animals were allowed only a maximum of 100 , or rarely 180 , training trials (including the 30 trials needed to reach criterion) before training was discontinued. Recalling that a decorticate rat may have a generalized performance deficit and also that the normal animal typically requires 25 to 30 trials to establish a brightness discrimination, a 100-trial cutoff would seem somewhat demanding. Thus, while it is obvious that Lashley's routine was sensitive to certain effects of visual decortication, whether these 
effects directly reflected an elevation in the animal's differential brightness threshold may be open to some question.

Because of this, and because of the importance of a possible dissociation of threshold capabilities following striate cortex lesions, it was decided to redetermine the differential brightness threshold of the normal and striate lesioned rat. To improve the sensitivity of the experiment, a titration procedure was used in an attempt to more critically establish threshold performance. In general, the data indicate that if one ignores an obvious and persistent general performance deficit, as Lashley did, the visual decorticate rat is indeed impaired in its ability to detect difference in brightness. However, if one takes into account this general performance deficit, then the visual decorticate rat's ultimate performance is quite similar to that of a normal rat and it would appear that the differential brightness threshold, like the absolute brightness threshold, is not directly dependent upon the integrity of the visual neocortex.

\section{METHOD}

\section{Subjects}

The subjects were six male hooded rats between 90 and 110 days of age at the beginning of the experiment. Throughout the experiment, all subjects were housed individually in standard laboratory cages in a room in which the day-night cycle was reversed so that all training was conducted during the dark portion of the rat's "day." All subjects were provided free access to food and water.

\footnotetext{
Apparatus

The apparatus was a Yerkes Y-maze, consisting of a startbox, a choice area, and two goal compartments, with the start and choice areas having a shock grid floor (see LeVere \& Morlock, 1973, 1974, for details). The goal compartments were separated from the choice compartment by clear plastic doors, and distinguished by transilluminated rear walls. The illumination of each rear wall was provided by a $150-\mathrm{W}$ flood lamp located some $70 \mathrm{~cm}$ behind it. The lamp was powered by a voltage adjusted to yield a brightness of $100 \mathrm{fL}$ (Tektronics digital photometer Model J-16 and a J-6503 probe) at the level of the rat's eye $.5 \mathrm{~cm}$ from the closed entrance door of the goal compartment. Lower brightnesses for discrimination training and titration testing were obtained by interposing apertures of different diameters $2.5 \mathrm{~cm}$ in front of the flood lamps which produced a decreased, but even, illumination over the translucent rear wall without affecting the spectral characteristics of the discriminative cues.

The rats were trained and tested in this apparatus by being placed in the start compartment, following which the guillotine door separating it from the choice area was raised. The rat was allowed $10 \mathrm{sec}$ to exit the start compartment before a pulsating shock (approximately $1 \mathrm{sec}$ on $.5 \mathrm{sec}$ off) was initiated; the shock continued until the rat entered the choice area. Once in the choice area, the rat was allowed an additional shock-free 10 -sec period to complete a choice response before the pulsating shock was once again initiated. Choice responses were accomplished by pushing down the clear plastic door to gain entrance to the goal compartment. The clear plastic door to the incorrect goal compartment was always locked, and any attempt to open it auto-
}

matically resulted in immediate and continuous footshock until the animal corrected its error. Following a correct choice, the rat was allowed approximately $15 \mathrm{sec}$ within the correct goal compartment and then returned to the transport cage to await the next trial.

\section{Procedure}

After handling, habituation to the discrimination box, and shaping, each rat was trained on a brightness discrimination with a 1 log unit difference between the correct and incorrect discriminative cues. For one group of three animals, the correct cue was $1 \mathrm{fL}$ and the incorrect cue was $10 \mathrm{fL}$, while for the other group of three animals, the correct cue was $100 \mathrm{fL}$ and the incorrect cue was $10 \mathrm{fL}$. Since it was desired that the animals be completely adapted to the testing procedures before attempting any threshold measurements, each subject was trained for $\mathbf{3 0}$ trials per day for 7 days each week until attaining a criterion of 5 successive days where performance was equal to or better than $90 \%$ first-choice responses.

On the day following attainment of this criterion, each animal was trained under the titration procedure to determine its differential brightness threshold. The titration routine was quite standard and involved an unchanging correct cue and a variable incorrect cue. The unchanging correct cue was the same brightness as the correct cue of the just completed discrimination problem, while the incorrect cue was initially some $.975 \log$ units differentbrighter in the case of the $1-\mathrm{fL}$ correct cue and dimmer in the case of the $100-\mathrm{fL}$ correct cue. Following trials in which the rat made a correct choice, the difference between the correct cue and the incorrect cue was decreased by an amount equal to .05 log units. Following trials in which the rat made an incorrect choice, the brightness difference was increased by $.05 \mathrm{log}$ units unless such an increase would result in a brightness difference greater than $.975 \mathrm{log}$ units. Thus, there was a progression of 19 different incorrect cues to the most difficult discrimination represented by a brightness difference of $.025 \mathrm{log}$ units between the correct and incorrect cues. Using this titration procedure, both groups of animals were tested for 50 trials per day until their daily mean performance and associated standard deviation stabilized with $\pm .05 \log$ units and $\pm .15 \log$ units, respectively, calculated according to the suggestion of Dixon and Massey (1951) (also see Rosenberger, 1970) for 7 successive days.

Throughout titration testing, the animals were dark-adapted in the experimental room for $30 \mathrm{~min}$ prior to testing, where the illumination measured at their transport cage was $.1 \mathrm{fL}$. In addition, they were returned to their transport cage for precisely $1 \mathrm{~min}$ between each discrimination trial.

Within $24 \mathrm{~h}$ following stabilization on the titration procedure, each animal sustained destruction of its visual neocortex. The ablation was performed by aspiration under sodium pentobarbital anesthesia and followed a line beginning at bregma and becoming tangential to the rhinal sulcus. Extreme care was exercised to assure removal of all neocortex posterior to this boundary. There were no animals which underwent sham operations, because it has been our experience that in our brightness training situation this operation has failed to produce any distinction relative to normal animals.

Fourteen days subsequent to the posterior neocortical lesion, the rats were returned to the Yerke's discrimination box and retrained in a manner identical to the preoperative procedure. That is, they were initially trained on the brightness discrimination involving discriminative cues differing in brightness by 1 log unit and following criterion performance on this problem were retrained on the titration procedure to determine their postoperative differential brightness thresholds. The sole exception to this was that if an animal failed to stabilize on the titration test within twice the number of trials required preoperatively, the animal's training was discontinued. 


\section{RESULTS}

\section{Histology}

Figure 1 presents dorsal surface diagrams of the extent of cortical destruction as well as frontal sections of the dorsal lateral geniculate nucleus for each animal. The darkened areas represent cortical destruction and retrograde cellular degeneration. In all cases, the lesions were what was intended and resulted in greater than $40 \%$ destruction of the rat's neocortex. For evaluation of retrograde degeneration within the dorsal lateral geniculate nucleus, these brains were embedded in plastic and sectioned at 25 microns, with every fourth section being saved and stained by cresyl violet. Examination of these sections indicated that in each animal there was complete retrograde degeneration of the dorsal lateral geniculate nucleus. Moreover, the sections indicated that the lesions themselves were restricted to neocortex and did not invade hippocampus or thalamic structures.

\section{Behavior}

Since none of the animals were able to postoperatively stabilize on the titration procedure, even when allowed between 1,150 and 3,300 trials (twice that required for preoperative stabilization), the overall mean performance computed according to Dixon and Massey (1951) was calculated for the 7 preoperative stabilization days and the last 7 days of postoperative testing. This estimate of the differential brightness threshold yielded a value of approximately $.13 \log$ units (.13 with the dim cues and

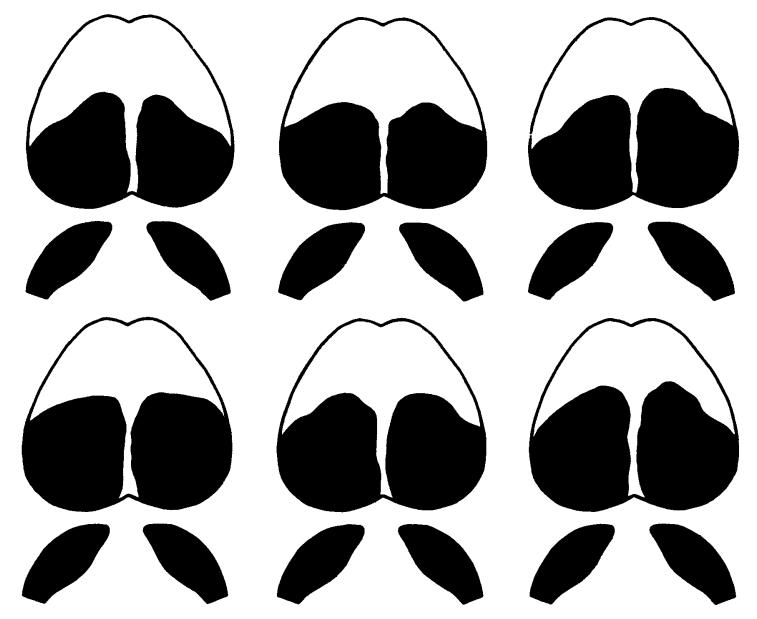

Figure 1. Dorsal surface diagrams of the neocortical destruction (black areas) for each subject of the present study. Below each diagram is an outline of the dorsal lateral geniculate nucleus at a level of $3.1 \mathrm{~mm}$ from the interaural line, with the blacked out area representing retrograde degeneration. The three animals represented in the top row responded to the dim discriminative cues, while the three animals represented in the bottom row responded to the bright discriminative cues.
.14 with the bright cues) for preoperative performance and approximately $.31 \mathrm{log}$ units (.26 with the dim cues and .36 with the bright cues) for postoperative performance. This increase in the differential brightness threshold following striate decortication is significant when tested with the $t$ statistic for correlated samples $(t=7.34$, df $=5)$. Thus, simply considering the brain-damaged rat's overall average performance on the titration test without regard for any general impairment which may accompany brain damage, Lashley's original conclusion would appear to be confirmed.

However, it must be remembered that the statistical procedure suggested by Dixon and Massey computes mean performance on the basis of failures, in the present case errors, which occur throughout the titration test. The assumption so far as threshold estimation is concerned is, of course, that each error is a direct result of the animal's attempt to detect a brightness difference below its threshold. But if the brain-damaged rat is hampered by a generalized performance impairment, as suggested by both Cooper et al. (1967) and Lashley (1930), then this assumption may be inappropriate. And, if all of the errors committed by the brain-damaged rat are not directly related to its ability to detect small differences in brightness, then any estimate of threshold based on overall mean performance will necessarily be inflated.

For example, consider Figures $2 \mathrm{~A}$ and $2 \mathrm{~B}$. These figures present the mean performance on each of the 50 trials of the titration test during the last 7 days of preoperative testing (solid line) and the last 7 days of postoperative testing (broken line). Figure $2 \mathrm{~A}$ is for the group of rats responding to the dim discriminative cues and Figure $2 \mathrm{~B}$ is for the group of rats responding to the bright discriminative cues. The vertical lines around alternating data points are the .05 confidence intervals. Inspection of these figures points out several relevant features of the destriate rat's discrimination behavior. First, the performance of the normal and of the brain-damaged rat is quite comparable over the final 10 trials of the titration test. In fact, on the basis of the .05 confidence intervals, there is no reason to consider them different. Second, this terminal performance for both the normal and lesioned rat represents an ability to detect brightness differences on the order of .1 to $.15 \mathrm{log}$ units. For the normal rat, this is similar to the previously estimated $.13 \mathrm{log}$ unit differential brightness threshold as calculated from the overall mean performance. However, for the rat with a visual lesion, this ability is far better than the $.31 \log$ unit differential brightness threshold estimated from the animal's overall mean performance. Clearly, the destriate rat's mean performance on the titration test is not a true reflection of its ability to detect small brightness differences. Finally, even though the destriate rat is extremely well trained on the experi- 
mental procedure, it nonetheless commits a number of errors on the initial trials of the titration test when the brightness differential is well above the threshold. This is in contradistinction to the normal rat which performs flawlessly until reaching its threshold limit. Taken together, these data indicate that the most salient difference between the destriate rat and its normal counterpart is the general facility with which the titration procedure is completed. However, the effects of striate ablation on the animal's differential brightness threshold is somewhat less clear.

The problem in making this determination is, of course, to obtain a relatively unbiased estimate of the destriate rat's differential brightness threshold. Clearly, the existence of a general impairment associated with the neurological insult renders the overall mean invalid. Performance on the final few trials of the titration test (see Figures $2 \mathrm{~A}$ and $2 \mathrm{~B}$ ) may provide a more reasonable estimate, but this measure is also confounded by any general impairment which may exist. What is necessary is an estimate of the destriate rat's differential brightness threshold which corrects for errors not directly related to threshold performance. While no correction procedure is totally unequivocal, the nature of any general impairment and the titration itself allow one approach which seems to have some degree of validity. This approach takes advantage of two facts. The first is that, while errors caused by any general impairment may occur throughout the titration test, errors caused by discriminations attempted below threshold can only occur when the brightness differential is below threshold. The second is that threshold performance occurs only during the latter trials of the titration test. This is because the titration run starts with a number of discrimination problems well above threshold and decreases the brightness differential between the cues only when the animal responds correctly. Therefore, a number of trials will occur early in the titration test when the rat is necessarily discriminating brightness differences above threshold. It would thus seem to follow that incorrect choice responses during these trials should reflect performance impairments not directly related to the animal's threshold. Moreover, any differences between the performance of the normal rat and the destriate rat on these early trials should provide an estimate of any general impairment which may result from the cortical ablation.

Following this line of thought, we used the follow-
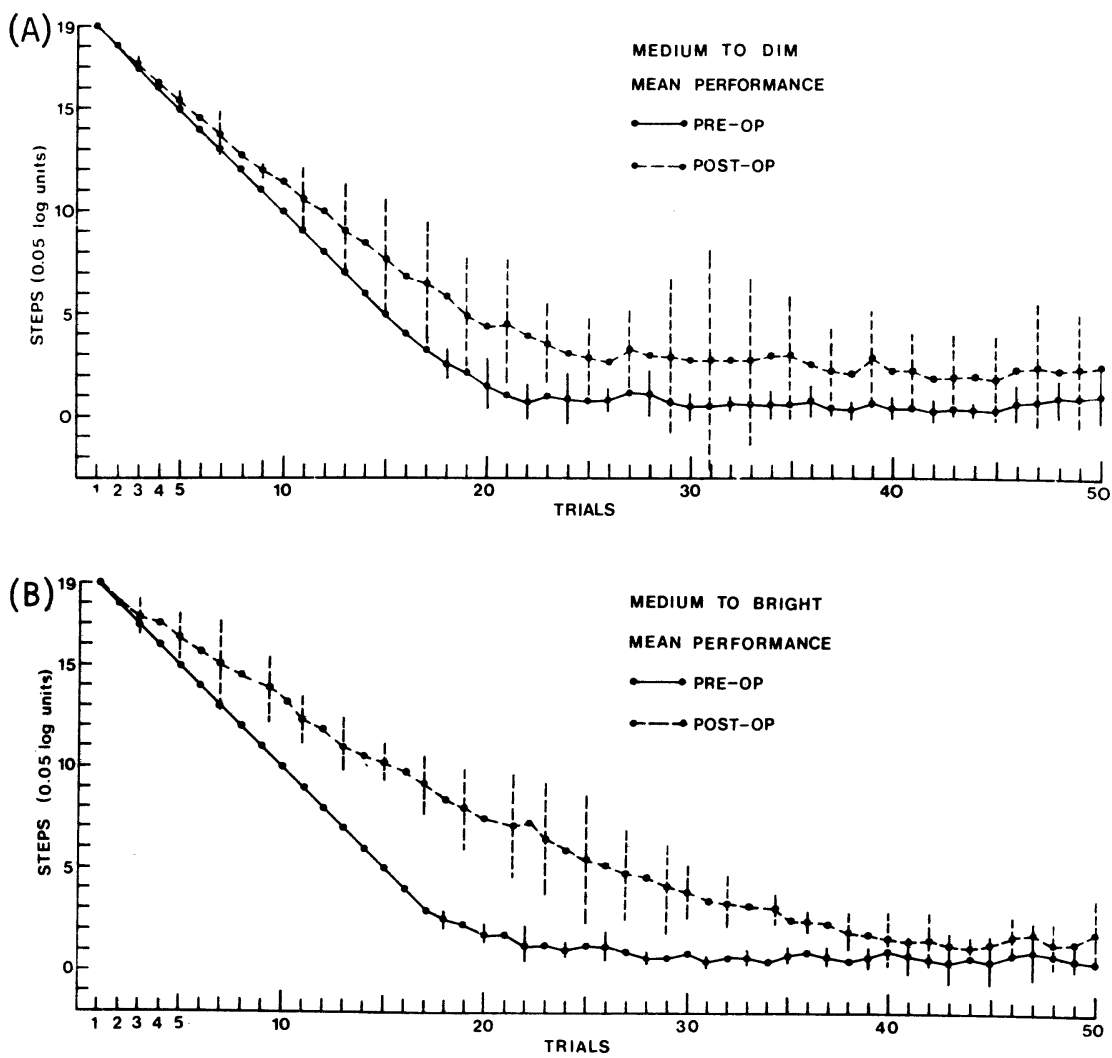

Figure 2. Mean performance over the final 7 days of titration testing for the normal animals (solid line) and the striate-lesioned animals (dashed line). In Figure $2 A$, the animals were trained with the 1-fL cue as the correct cue, while in Figure $2 \mathrm{~B}$, the animals were trained with the 100 -fL cue as correct. The vertical bars around alternating points represent .05 confidence intervals. 
ing formula to correct the estimate of the striate rat's threshold performance both in terms of errors committed and in terms of its ability to detect small differences in brightnesses:

$\begin{array}{ll}\begin{array}{l}\text { Corrected threshold } \\ \text { performance trials: } \\ 41-50\end{array} & \begin{array}{l}\text { Postoperative } \\ \text { performance: } \\ \text { trials 41-50 }\end{array} \\ & -\left(\begin{array}{l}\text { Postoperative } \\ \text { performance: }-\begin{array}{l}\text { Preoperative } \\ \text { performance: } \\ \text { trials 1-10 }\end{array}\end{array}\right)\end{array}$

We chose the initial 10 trials of the titration test as the basis for this correction because, by definition, the minimal brightness which may occur is $.525 \mathrm{log}$ unit and this difference is well above any estimate of the striate rat's threshold. The final 10 trials of the titration test were selected to estimate threshold performance principally on the basis of inspection of the curves presented in Figures 2A and 2B.

Considering first the error scores of the normal and the visual decorticate rat, the normal rat progresses through the first 10 trials of the titration test without fault while the lesioned rat averages 1.3 errors. Because there is no variance associated with the normal rat's mean, this difference is, of course, significant. During the final 10 trials of the titration test, the normal rat commits an average of 3.6 errors while the corresponding error score for the destriate rat is 4.3 errors. Using the $t$ statistic for correlated samples, the difference between these two error scores is significant at the .05 level $(t=2.752$, $\mathrm{df}=5$, two-tailed). However, if the destriate rat's performance over these final 10 trials is corrected as suggested above, then, the mean error score drops to 3.0 which is not significantly different from the mean errors committed by the normal animals $(t=1.460$, $\mathrm{df}=5$, two-tailed).

Turning to the brightness differences which may be detected by the normal and striate-lesioned rat, a similar pattern of results emerges. That is, the mean $\log$ brightness difference detected over the initial 10 trials by the normal rat is .750 , while that for the visual decorticate rat is $\mathbf{8 0 1}$. Although this difference is significant, the significance is not altogether meaningful since, as noted above, the normal animal makes no errors during these initial trials and there is thus zero variability associated with its mean performance. With regard to the mean brightness difference detected by the normal and destriate rat during the final 10 trials of the titration test, the respective $\log$ values are .058 and .118 . Like the error scores, this difference is significant $(t=3.857, \mathrm{df}=5$, $\mathrm{p}<.02$, two-tailed). But, if the destriate rats' performance is corrected to account for a generalized impairment, then the brightness difference drops to $.066 \mathrm{log}$ units and this value is not significantly different from that obtained by the normal animal $(t=0.37$, df $=5$, two-tailed). Thus, if one corrects for the general impairment associated with neocortical destruction, one finds that the threshold performance of the visual decorticate is identical to that of the normal rat in terms of both errors and the ultimate brightness difference it is able to detect.

\section{DISCUSSION}

The present research was designed to address a single question: Is the differential brightness threshold in rat dependent upon the integrity of the primary visual neocortex? The question was considered important for two principal reasons. First, even though striate cortex lesions may severely disrupt the performance of a learned brightness discrimination in the rat, certain aspects of this learning are apparently spared (LeVere, 1975; LeVere \& Morlock, 1973, 1974). If certain aspects of the preoperative learning, notably those characterized as associations or engrams, may be spared following visual neodecortication, might not the animals' more fundamental psychophysical response to light be also spared? Second, previous data has suggested a dissociation of psychophysical functions following visual cortex damage. For example, Cooper et al. (1967) has indicated that the absolute brightness threshold is unaffected by striate lesions. On the other hand, Lashley (1930) has argued that the differential brightness threshold is significantly elevated following visual ablation. If both of these results are valid, then the data offers a most important proposition. This is that, while certain psychophysical thresholds may not depend upon the neocortex, others may be highly dependent upon the neocortex.

However, Lashley's conclusions may be questioned on the grounds that, while he reported a general performance impairment, he did not consider this in his determination of the lesioned rat's differential brightness threshold. Clearly, Lashley's rats were impaired on visual discrimination tasks, as were the animals of the present study and those tested by Cooper et al. (1967). But before this impairment may be considered as a change in threshold, one must adequately assess performance abilities and attempt to separate these from threshold capabilities. For example, the overall mean performance of the brain-damaged rats on the titration test used in the present research was significantly higher than that of the normal animal. If the striate rat is unable to detect brightness differences as small as those detectable by the normal rat, then this increase in mean performance may indeed reflect a shift in threshold. But, if one supposes that the striate rat suffers from some general impairment not directly related to threshold discrimination performance, then this increase in mean performance may not reflect a shift in the 
animal's differential brightness threshold. On the basis of the present results, we believe that the performance impairment exhibited by rats lacking visual cortex is a general one and does not reflect a change in threshold capabilities.

There are three aspects of the present data which support this belief. First, if one considers the ultimate brightness differences the destriate rat is able to detect on the titration test, then there is very little difference between the brain-damaged animal and its normal counterpart. Moreover, it must be remembered, that it is these terminal test trials which best reflect threshold capabilities under the titration procedure.

Second, the overall mean performance of the normal rat is quite similar to its performance at the conclusion of the titration test. In distinction to this, the overall mean performance of the brain-damaged animal is much higher than its performance at the conclusion of the titration test. This indicates that the destriate rat, unlike the normal rat, may commit a number of errors unrelated to threshold discriminations, and if these errors are included in the estimate of threshold, then the estimate will, by definition, be spuriously high. In other words, while the overall mean performance may be a satisfactory estimate of threshold in the normal rat, it is probably invalid for the brain-damaged rat, because it includes errors not related to threshold discrimination performance. On the other hand, if it is assumed that all of the errors committed by the lesioned rat are the result of a shift in threshold, then this shift would have to be on the order of nearly $1 \mathrm{log}$ unit, since the destriate rat responds incorrectly at the very beginning of the titration test. While the effects of visual neodecortication may be severe, a shift in threshold of this magnitude is highly unlikely.

Third, and most importantly, if one uses the destriate rat's performance above threshold discrimination performance to correct its terminal performance on the titration test, then there is virtually no difference between the normal rat and the destriate rat. In quantitative terms, the mean brightness differential detectable by the normal rat is $.058 \log$ units, while the corrected mean brightness differential detectable by the brain-damaged rat is $.067 \mathrm{log}$ units-a difference of only $.009 \mathrm{log}$ units, which is clearly trivial by any criterion.

There is, however, one final procedural point which must be considered before one can accept the proposition that the differential brightness threshold is not dependent upon the integrity of the primary visual neocortex. This point concerns the possibility that the step size of the present titration procedure was too large and thus created a floor which prevented the rat from attaining its true threshold limit. That is, if the smallest brightness difference of the titration test was above threshold for the normal rat and at or above threshold for the destriate rat, then their performances, as measured by the titration procedure would, by definition, be the same. To check this possibility, we calculated the percentage of correct responses on those discriminations involving the smallest brightness differential for both the normal and the brain-damaged rat. If this percentage was significantly above chance-above $75 \%$ if one adheres to classic psychophysical traditionthen the present titration test was probably compromised by a floor effect. However, this does not seem to be the case, because the normal animals only responded correctly on $57 \%$ of these discriminations while the destriate animals responded correctly on only $54 \%$. Neither of these values deviates from what would be expected by chance. Thus, while the visual decorticate rat is, in terms of discriminating brightnesses, anything but normal, this abnormality does not appear to reflect a change in the differential brightness threshold.

Speculating on what this abnormality might be, it is possible to suggest that while the neocortex may not be directly involved in the threshold capacities of the rat, it nonetheless may be involved in the utilization of these capacities. This notion, while not as rigorous as one would like, is, however, not without precedence. For example, Meyer (1972) has suggested that performance of a learned habit involves not only the storage of memory engrams but also the accessing of these engrams. The storage of engrams, it is further argued, is very stable (Meyer $\&$ Beattie, in press), so the deficits associated with neocortical damage may be a dysfunction of the accessing process (LeVere, 1975). In the present case, we would only suggest that the term "accessing" be replaced by the term "utilization," since we here are dealing with psychophysical functions and not learned engrams. From a different tack, but one somewhat more akin to the present results, Braun and his associates (Braun, Slick, \& Lorden, 1972; Wolf, Dicara, \& Braun, 1970; Braun, Note 1) have demonstrated that lesions of gustatory neocortex do not disrupt taste thresholds per se but rather the animal's ability to utilize that stimuli in a learning task. This is, of course, exactly the conclusion reached by Cooper et al. (1967) in their evaluation of absolute brightness thresholds and the conclusion of the present research concerned with respect to differential brightness thresholds. Furthermore, in a recent review of learning capacities, Berlucchi and Buchtel (1975) have suggested that more often than not it is efficiency which is impaired following neocortical brain damage and not the ultimate ability to establish conditioned and operant responses. 


\section{REFERENCE NOTE}

1. Braun, J. J. Preference-aversion functions for the basic taste stimuli in rats lacking gustatory neocortex. Paper presented at meetings of the Psychonomic Society, Denver, 1975.

\section{REFERENCES}

BerluCCHI, G., \& Buchtel, H. A. Some trends in the nuerological study of learning. In M. S. Gazzaniga \& C. Blakemore (Eds.), Handbook of psychobiology. New York: Academic Press, 1975.

BraUN, J. J., SLICK, T. B., \& LORDEN, J. F. Involvement of gustatory neocortex in the learning of taste aversions. Physiology and Behavior, 1972, 9, 637-641.

Cooper, R. M., Freeman, I., \& Pinel, J. P. J. Absolute threshold of vision in the rat after removal of striate cortex. Journal of Comparative and Physiological Psychology, 1967, 64, 36-39.

DiXoN, W. J., \& MAsSEY, F. J. Introduction to statistical analysis. New York: McGraw-Hill, 1951.

LASHLEY, K. S. The mechanism of vision: II. The influence of cerebral lesions upon the threshold of discrimination for brightness in the rat. Journal of Genetic Psychology, 1930, 37, 461-480.

LASHLEY, K. S. The mechanism of vision: XII. Nervous structures concerned in habits based on reactions to light. Comparative Psychology Monographs, 1935, 11, 43-79.
LEVERE, T. E. Neural stability, sparing, and behavioral recovery following brain damage. Psychological Review, 1975, 82, 344-358.

LEVERE, T. E., \& MORLOCK, G. W. The nature of visual recovery following posterior neodecortication in the hooded rat. Journal of Comparative and Physiological Psychology, 1973, 83, 62-67.

LEVERE, T. E., \& MORLOCK, G. W. The influence of preoperative learning on the recovery of a successive brightness discrimination. Bulletin of the Psychonomic Society, 1974, 4, 507-509.

Meyer, D. R. Access to engrams. American Psychologist, 1972, 27, 124-133.

MEYER, D. R., \& BEATtie, M. S. The engrams of habits are formed very rapidly, and then become almost immediately resistant to changes as a function of time. In L. Miller, C. A. Sandman, \& A. Kastin (Eds.), Neuropeptide influences on brain and behavior mechanisms. New York: Raven Press, in press.

ROSENBERGER, P. B. Response-adjusting stimulus intensity. In W. S. Stebbins (Ed.), Animal psychophysics: The design and conduct of sensory experiments. New York: Appleton-CenturyCrofts, 1970.

Wolf, G., Dicara, L. V., \& Braun, J. J. Sodium appetite in rats after neocortical ablation. Physiology and Behavior, 1970, 5. 1265-1269.

(Received for publication April 4, 1977; revision accepted October $11,1977$. 